Article

\title{
Comparative Evaluation of the Photostability of Carbamazepine Polymorphs and Cocrystals
}

\author{
Reiko Yutani ${ }^{1, *}$, Ryotaro Haku ${ }^{2}$, Reiko Teraoka ${ }^{1,3, *}$, Chisato Tode ${ }^{4}$, Tatsuo Koide ${ }^{5}$, \\ Shuji Kitagawa ${ }^{6}$, Toshiyasu Sakane ${ }^{1}$ and Toshiro Fukami ${ }^{2, *}$ \\ 1 Laboratory of Pharmaceutical Technology, Kobe Pharmaceutical University, 4-19-1 Motoyamakita-machi, \\ Higashinada-ku, Kobe 658-8558, Japan \\ 2 Department of Molecular Pharmaceutics, Meiji Pharmaceutical University, 2-522-1 Noshio, Kiyose, \\ Tokyo 204-8588, Japan \\ 3 Department of Pharmaceutical Technology, College of Pharmaceutical Sciences, Himeji Dokkyo University, \\ 7-2-1 Kamiohno, Himeji, Hyogo 670-8524, Japan \\ 4 Instrumental Analysis Center, Kobe Pharmaceutical University, 4-19-1 Motoyamakita-machi, \\ Higashinada-ku, Kobe 658-8558, Japan \\ 5 Division of Drugs, National Institute of Health Sciences, 3-25-26 Tonomachi, Kawasaki-ku, Kawasaki, \\ Kanagawa 210-9501, Japan \\ 6 Kobe Pharmaceutical University, 4-19-1 Motoyamakita-machi, Higashinada-ku, Kobe 658-8558, Japan \\ * Correspondence: r-yutani@kobepharma-u.ac.jp (R.Y.); teraoka@gm.himeji-du.ac.jp (R.T.); \\ fukami@my-pharm.ac.jp (T.F.); Tel.: +81-78-441-7532 (R.Y.); +81-79-223-6833 (R.T.); +81-42-495-8936 (T.F.)
}

Received: 24 September 2019; Accepted: 22 October 2019; Published: 24 October 2019

\begin{abstract}
Carbamazepine (CBZ), a widely used antiepileptic, is known to be sensitive to light. The aim of this study was to evaluate the photostabilities of three cocrystals of CBZ (CBZ-succinic acid (SUC), CBZ-saccharin (SAC) form I, and CBZ-SAC form II) illuminated with a $\mathrm{D}_{65}$ fluorescent lamp compared with those of the conventional solid forms: CBZ polymorphs (forms I, II, and III). The order of discoloration determined using a colorimetric measurement was almost consistent with that of the degradation rates estimated using Fourier-transform infrared reflection-absorption spectroscopy, and these parameters of CBZ polymorphs increased in the order of form III, form I, and form II. CBZ-SUC and CBZ-SAC form I significantly suppressed the discoloration and degradation of CBZ compared with the raw CBZ, while CBZ-SAC form II facilitated the discoloration and degradation of CBZ. These results were supported by the results from the low-frequency Raman spectroscopy. The molecular mobility estimated using solid-state nuclear magnetic resonance ${ }^{1} \mathrm{H}$ spin-lattice relaxation time strongly correlated with the degradation rate constant, indicating that molecular mobility significantly decreased following the formation of CBZ-SUC and CBZ-SAC form I and resulted in higher photostability. Overall, CBZ-SUC and CBZ-SAC form I are photostable forms and cocrystallization was proven to be an effective approach to improving the photostability of a photolabile drug.
\end{abstract}

Keywords: carbamazepine; photostability; polymorphs; cocrystal; succinic acid; saccharin

\section{Introduction}

The efficacy, safety, and quality of pharmaceuticals are adversely affected by light exposure. Most drugs are exposed to light during production, storage, distribution, handling, and use $[1,2]$. Light potentially alters physicochemical properties of the active pharmaceutical ingredients (APIs) and affects the stability of the final products [1-3]. Light can provoke the photodecomposition of a drug, which may cause not only a loss of potency of the drug but also the production of a highly toxic compound. Thus, a better understanding of the nature and extent of photodecomposition, 
the mechanisms of the degradation, and the wavelength causing instability is needed to stabilize the product $[2,3]$.

Carbamazepine (CBZ), a widely used antiepileptic, is known to be a photosensitive drug and the form issued by the manufacturer has been reported to exhibit a color change after 3 days of illumination with a xenon lamp. Form diversity of CBZ is also well known, and many reports have examined its polymorphs [4,5], hydrate and solvate forms [5,6], and the relationship between its solid form and physicochemical properties $[7,8]$.

Recently, cocrystals have received considerable attention as a new class of API solids. A cocrystal is defined as a multiple crystalline molecular complex coexisting through noncovalent interactions such as hydrogen bonding, $\pi-\pi$ stacking interactions, and van der Waals forces $[9,10]$. Cocrystals can alter the physicochemical properties of the API without modifying its intrinsic structure [10]. In addition, the cocrystallization technique has been applied to a wide range of drugs because it does not require proton transfer between components, unlike salt formation [11]. CBZ has been shown to form cocrystals with various cocrystal formers (coformers), including dicarboxylic acid and saccharin (SAC) [12]. Favorable physicochemical properties of these cocrystals have been reported previously. Ullah et al. reported the advantages of a cocrystal of CBZ with succinic acid (SUC) (CBZ-SUC) in terms of aqueous solubility, in vitro drug release, and bioavailability [13]. According to Hickey et al., a cocrystal of CBZ with SAC (CBZ-SAC form I) potentially represents a viable alternative to the conventional anhydrous polymorph due to its physical and chemical stability and oral bioavailability [14]. As shown in a study by Porter III et al., a polymorph exists in a cocrystal of CBZ with SAC (CBZ-SAC form II) [15]. Although many reports have analyzed CBZ cocrystals, no studies have investigated their photostabilities. In addition, fewer studies have focused on the photosensitivity of cocrystals compared with those concentrated on the conventional solid forms [16-19].

Thus, in the present study, we prepared two polymorphs (CBZ form I and form II) and three cocrystals of CBZ (CBZ-SUC, CBZ-SAC form I, and CBZ-SAC form II) and compared their photostabilities with CBZ form III, which is known to be a comparatively stable form of CBZ. Photostability was assessed using colorimetric measurements, low-frequency Raman spectroscopy, Fourier-transform infrared reflection-absorption spectroscopy (FT-IR RAS) and solid-state nuclear magnetic resonance (SSNMR) ${ }^{1} \mathrm{H}$ spin-lattice relaxation time $\left(\mathrm{T}_{1}\right)$.

\section{Materials and Methods}

\subsection{Materials}

CBZ (purity: $\geq 97.0 \%$ ) was purchased from Fujifilm Wako Pure Chemical Corporation (Osaka, Japan). SUC (purity: $\geq 99.5 \%$ ) and SAC (purity: $\geq 98.0 \%$ ) were purchased from Nacalai Tesque, Inc. (Kyoto, Japan). The chemical structures of CBZ, SUC, and SAC are shown in Figure 1. All the other reagents were of analytical grade or reagent grade.<smiles>NC(=O)N1c2ccccc2C=Cc2ccccc21</smiles>

(a)<smiles>O=C(O)CCC(=O)O</smiles>

(b)<smiles>O=C1NS(=O)(=O)c2ccccc21</smiles>

(c)

Figure 1. Chemical structures of (a) carbamazepine (CBZ), (b) succinic acid (SUC), and (c) saccharin (SAC).

\subsection{Preparation of $C B Z$ Polymorphs}

CBZ polymorphs (forms I and II) were prepared according to a previously reported method [20]. 
CBZ form I: $\mathrm{CBZ}$ was dissolved in a $50 \%$ ethanol solution at $70{ }^{\circ} \mathrm{C}$, stored at room temperature and dried in vacuo for $3 \mathrm{~h}$, obtaining CBZ-dihydrate. Then, CBZ form I was obtained by drying CBZ-dihydrate in vacuo at $115^{\circ} \mathrm{C}$ for $6 \mathrm{~h}$.

CBZ form II: CBZ was dissolved in chloroform, after which diethyl ether was added and stirred at room temperature. The precipitated crystals were then filtered and dried in vacuo in a desiccator containing $\mathrm{P}_{2} \mathrm{O}_{5}$ at room temperature for $3 \mathrm{~h}$, obtaining CBZ form II.

CBZ form III: The commercial product was used without further purification.

\subsection{Preparation of CBZ Cocrystals}

Three cocrystals were prepared according to previously reported methods [13-15].

For the preparation of CBZ-SUC, $1.00 \mathrm{~g}$ of CBZ and $0.25 \mathrm{~g}$ of SUC (molar ratio: 2:1) were dissolved in $30 \mathrm{~mL}$ of ethanol at $70{ }^{\circ} \mathrm{C}$ and stored at room temperature. The precipitated crystals were then filtered and dried in vacuo at room temperature.

For the preparation of CBZ-SAC form I, $1.04 \mathrm{~g}$ of CBZ and $0.81 \mathrm{~g}$ of SAC (molar ratio: 1:1) were dissolved in $20 \mathrm{~mL}$ of ethanol, stored overnight at $50^{\circ} \mathrm{C}$ and then maintained at $30^{\circ} \mathrm{C}$. The precipitated crystals were filtered and dried in vacuo at room temperature.

For the preparation of CBZ-SAC form II, $1.04 \mathrm{~g}$ of CBZ and $0.81 \mathrm{~g}$ of SAC (molar ratio: 1:1) were dissolved in $20 \mathrm{~mL}$ of ethanol at $70{ }^{\circ} \mathrm{C}$ and stored overnight at room temperature. The precipitated crystals were filtered and dried in vacuo at room temperature.

As a control, physical mixtures (PMs) of CBZ form III and SUC or SAC were prepared by weighing $\mathrm{CBZ}$ and each coformer in an equivalent molar ratio to cocrystal and mixing them with a vortex mixer.

\subsection{Powder X-Ray Diffractometry (PXRD)}

PXRD patterns of CBZ polymorphs, coformers, PMs, and cocrystals were recorded using an X-ray diffractometer (RINT-Ultima, Rigaku Co., Tokyo, Japan) with $\mathrm{CuK} \alpha$ radiation generated at $36 \mathrm{kV}$ and $20 \mathrm{~mA}$ at room temperature. Data were collected within a diffraction angle range of $5^{\circ}-40^{\circ}(2 \theta)$ at a step size of $0.02^{\circ}$ and a scanning speed of $2^{\circ} / \mathrm{min}$.

\subsection{Thermal Analysis}

Differential scanning calorimetry (DSC) analyses were performed using a DSC 3500 Sirius instrument (NETZSCH Japan K.K., Yokohama, Japan). Two milligrams of each sample were weighed in an aluminum pan and heated from 30 to $240{ }^{\circ} \mathrm{C}$ at a rate of $10.0^{\circ} \mathrm{C} / \mathrm{min}$.

\subsection{Colorimetric Measurement}

One hundred milligrams of CBZ polymorph, PMs, and cocrystal powders were compressed to prepare tablets with $10 \mathrm{~mm}$ in diameter using a compression/tension testing machine (TG-50kN, MinebeaMitsumi Inc., Tokyo, Japan) equipped with flat-faced punches and a cylindrical die. The compressed tablets were stored in a light-irradiation tester (Light-Tron LT-120, Nagano Science Co. Ltd., Osaka, Japan) equipped with a $\mathrm{D}_{65}$ fluorescent lamp. The illuminance was set to $3500 \mathrm{~lx}$. The irradiation tests were conducted at $25^{\circ} \mathrm{C}$.

The surface color of the tablet was measured with a color reader (CR-13, Konica Minolta Japan, INC., Tokyo, Japan) after the designated irradiation times. The color difference ( $\Delta E^{*}$ ab) before and after irradiation was calculated using Equation (1) to evaluate the degree of discoloration. All values were reported as the averages of three measurements:

$$
\Delta E_{\mathrm{ab}}^{*}=\sqrt{\left(L_{\mathrm{t}}^{*}-L_{0}^{*}\right)^{2}+\left(a_{\mathrm{t}}^{*}-a_{0}^{*}\right)^{2}+\left(b_{\mathrm{t}}^{*}-b_{0}^{*}\right)^{2}},
$$

where $\Delta E^{*}$ ab is the color difference, $L^{*}$ is the brightness, $a^{*}$ is the red/green coordinate, and $b^{*}$ is the yellow/blue coordinate. 
The apparent discoloration rate constant $(k)$ was calculated using Equation (2) [21]:

$$
\log \Delta E_{\mathrm{ab}}^{*}=\frac{1}{1-n} \log t+\frac{1}{1-n} \log [(1-n) k] .
$$

\subsection{Low-Frequency Raman Spectroscopy}

The CBZ form III, CBZ-SUC, or CBZ-SAC form II powder was ground using a mortal and a pestle, and then passed through a No. 200 sieve (nominal aperture size: $75 \mu \mathrm{m}$ ). The sieved powder was mixed with microcrystalline cellulose at a weight ratio of 1:9 (the sieved powder/ microcrystalline cellulose) for $5 \mathrm{~min}$. Two hundred milligrams of the resulting mixture were weighed and compressed to prepare tablets with a diameter of $8 \mathrm{~mm}$.

The sample tablets were irradiated with a $\mathrm{D}_{65}$ fluorescent lamp for $343 \mathrm{~h}$ (over 1.2 million $\mathrm{lx} \cdot \mathrm{h}$ ) as described above (Section 2.6). Qualitative analyses of changes in the surface of the tablets after irradiation were conducted using the Raman WorkStation (Kaiser Optical Systems Inc., MI, USA) equipped with a low-frequency XLF-CLM module (ONDAX Inc., CA, USA). The excitation wavelength was set to $976 \mathrm{~nm}$. The standard spectrum of each component was obtained with an exposure time of $10 \mathrm{~s}$ in three scans. Samples with an area of $500 \mu \mathrm{m} \times 500 \mu \mathrm{m}$ were scanned with a spectral range from -200 to $200 \mathrm{~cm}^{-1}$, a step size of $16.7 \mu \mathrm{m}$, and a resolution of $4.0 \mathrm{~cm}^{-1}$. The data were analyzed using ISys 5.0 software (Malvern Instruments Ltd., Worcestershire, UK). The spectral analysis was performed in a range of $5-200 \mathrm{~cm}^{-1}$. The spectrum of each component was preprocessed using the standard normal variate (SNV), and images were generated based on the standard spectrum of each API using the partial least squares (PLS) analysis.

\subsection{FT-IR RAS}

Five hundred milligrams of CBZ polymorph, PMs, and cocrystal powders were compressed to prepare tablets with a diameter of $20 \mathrm{~mm}$. The prepared tablets were fixed on a glass plate and irradiated with a $\mathrm{D}_{65}$ fluorescent lamp as described above (Section 2.6). FT-IR RAS spectra were obtained using a Frontier FT-IR system (PerkinElmer Japan Co., Ltd.). The spectra were modified by the Kramers-Kronig transform and baseline correction was performed. The remaining percentage was calculated from the intensity ratio of the $\mathrm{C}=\mathrm{O}$ stretching vibration (at $1675-1685 \mathrm{~cm}^{-1}$ ) of $\mathrm{CBZ}$ before and after irradiation [22]. The photodegradation rate constant was also calculated from the apparent first-order plots.

\subsection{SSNMR ${ }^{1} H$ Spin-Lattice Relaxation Time $\left(T_{1}\right)$}

Spin-lattice relaxation times $\left(\mathrm{T}_{1}\right)$ of $\mathrm{CBZ}$ polymorphs and cocrystals were measured using a Varian VXR ${ }^{1} \mathrm{H}$ SSNMR spectrometer (Varian Inc., Palo Alto, CA, USA) at $500 \mathrm{MHz}$. Cross-polarization and magic angle spinning (CP-MAS) methods were applied with a spinning rate of $20 \mathrm{kHz}$, a contact time of $200 \mu \mathrm{s}$, and a recycle delay of $600 \mathrm{~s}$, using a zirconia sample tube with a diameter of $3.2 \mathrm{~mm}$. Saturation recovery times were assayed from 0.1 to $1500 \mathrm{~s}$.

\section{Results}

\subsection{Characterization of CBZ Polymorphs and Cocrystals}

The PXRD patterns of CBZ polymorphs, two coformers, PMs, and cocrystals are shown in Figure S1 (Supplementary Materials). The PXRD patterns of CBZ form I, form II, and form III were consistent with the patterns reported by Grzesiak et al. (Figure S1a-c, Supplementary Materials) [4]. The PXRD patterns of CBZ-SUC, CBZ-SAC form I, and CBZ-SAC form II, which were also consistent with the patterns described in previous reports, were different from those of CBZ form III, the corresponding coformers, and PMs (Figure S1c-j, Supplementary Materials) [13-15]. 
The DSC profiles of CBZ polymorphs, two coformers, PMs, and cocrystals are shown in Figure S2 (Supplementary Materials). The DSC profiles of each polymorph were approximately consistent with the profiles described in a previous report (Figure S2a-c, Supplementary Materials) [4]. The DSC profiles of CBZ-SUC, CBZ-SAC form I, and CBZ-SAC form II exhibited the characteristic endothermic peaks at $191.7,177.9$, and $174.5^{\circ} \mathrm{C}$, respectively, which differed from those of CBZ form III, the corresponding coformers, and PMs (Figure S2c-j, Supplementary Materials). These results confirmed the formation of cocrystals.

\subsection{Changes in the Surface Color of Tablets}

Changes in the appearance of sample tablets were evaluated by calculating the difference in color before and after irradiation with a $\mathrm{D}_{65}$ fluorescent lamp and the apparent discoloration rate constant (k). Time courses for discoloration of CBZ polymorphs, PMs, and cocrystals as well as their apparent discoloration rate constants are shown in Figure 2 and Table 1, respectively. The surface color of tablets gradually turned from white to pale yellow-white following light exposure, and $\Delta E^{*}$ ab increased as the irradiation time increased. The discoloration rate constants of CBZ polymorphs increased in the order of form III, form I, and form II, and the surface color of the tablet of form II turned yellow. The surface color of the tablets of PMs also changed, similar to that of the raw CBZ. On the other hand, the discoloration rate constant of CBZ cocrystals differed. The discoloration rate constants of CBZ-SUC and CBZ-SAC form I were significantly lower than those of the raw CBZ and the corresponding PMs, that is, changes in appearance were significantly suppressed. Meanwhile, the discoloration rate constant of CBZ-SAC form II was significantly higher and the surface color of the tablet more quickly turned yellow.

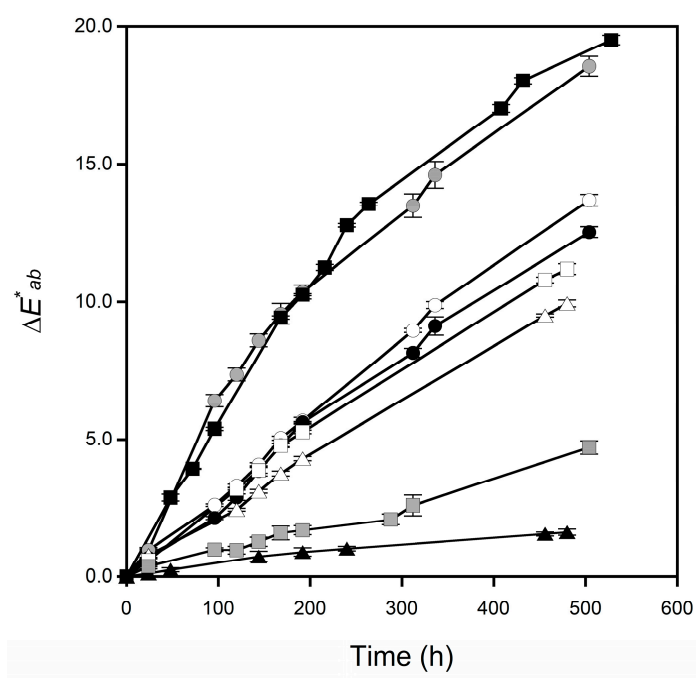

Figure 2. Time courses of the discoloration of CBZ polymorphs, physical mixtures (PMs), and cocrystals. The symbols represent changes in $\Delta E_{\mathrm{ab}}^{*}$ of CBZ form I $(\bigcirc)$, form II (๑), form III $(\bullet)$, CBZ-SUC PM $(\triangle)$,

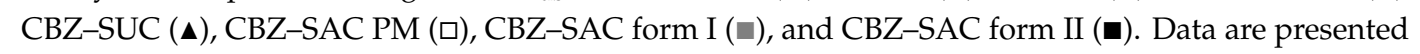
as the means $\pm \mathrm{SD}$ of 3 values.

Table 1. Apparent discoloration rate constants of CBZ polymorphs, PMs, and cocrystals.

\begin{tabular}{cc}
\hline Sample & Apparent Discoloration Rate Constant $\left(\mathbf{h}^{\mathbf{- 1}}\right)$ \\
\hline CBZ form I & 0.032 \\
CBZ form II & 0.054 \\
CBZ form III & 0.026 \\
CBZ-SUC PM & 0.024 \\
CBZ-SUC & 0.004 \\
CBZ-SAC PM & 0.026 \\
CBZ-SAC form I & 0.008 \\
CBZ-SAC form II & 0.064 \\
\hline
\end{tabular}




\subsection{Evaluation of Photodegradation on the Surface of Tablets Using Low-Frequency Raman Spectroscopy}

Photodegradation of the surface of sample tablets of CBZ form III, CBZ-SUC, and CBZ-SAC form II under a $\mathrm{D}_{65}$ fluorescent lamp was evaluated using low-frequency Raman spectroscopy. Low-frequency Raman spectroscopy is a simple and highly sensitive analytical technique for identifying the crystal form $[23,24]$. Standard spectra of CBZ form III, CBZ-SUC, CBZ-SAC form II, and microcrystalline cellulose are shown in Figure S3 (Supplementary Materials). Blue-to-red domains in each image represent the low-to-high density of each API. For CBZ form III and CBZ-SUC (Figure 3a,b), the Raman images and representative spectra of each domain before and after $343 \mathrm{~h}$ of irradiation were not substantially different, indicating that these crystal forms were comparatively photostable. In contrast, the blue domain of CBZ-SAC form II increased and the characteristic peaks of the cocrystal in the domain almost disappeared after $343 \mathrm{~h}$ of irradiation (Figure 3c), indicating that part of CBZ-SAC form II photodegraded after irradiation.
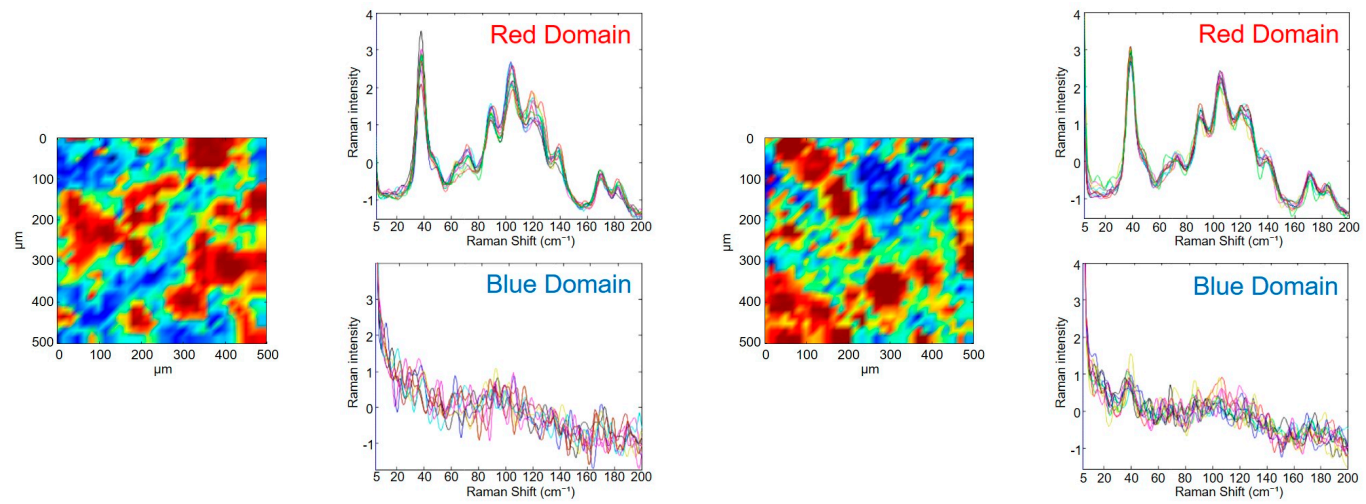

(a)
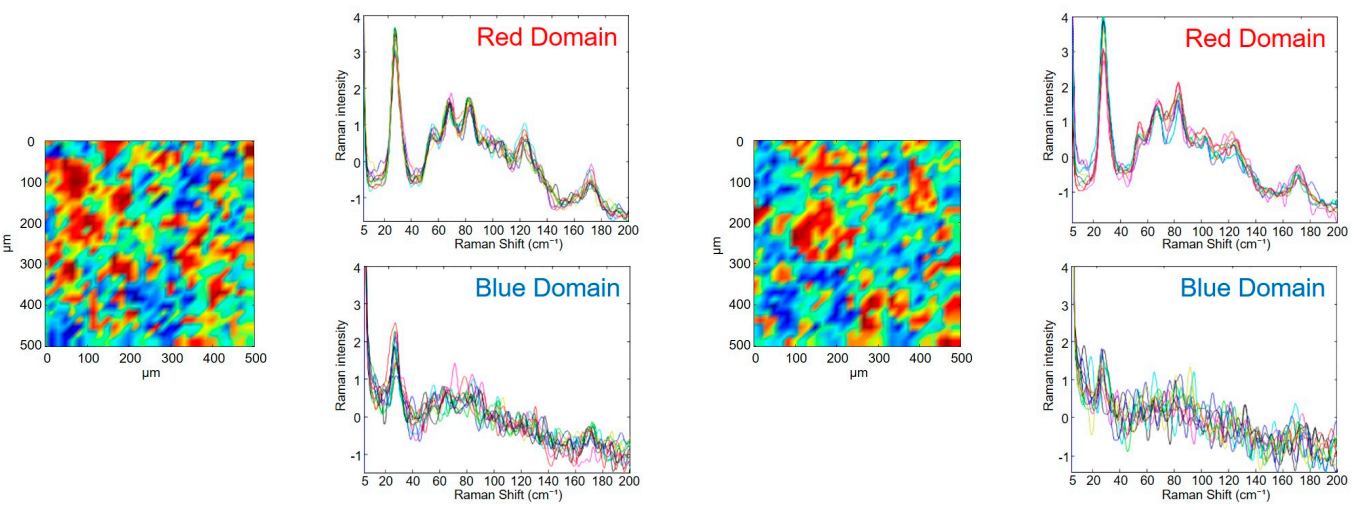

(b)

Figure 3. Cont. 

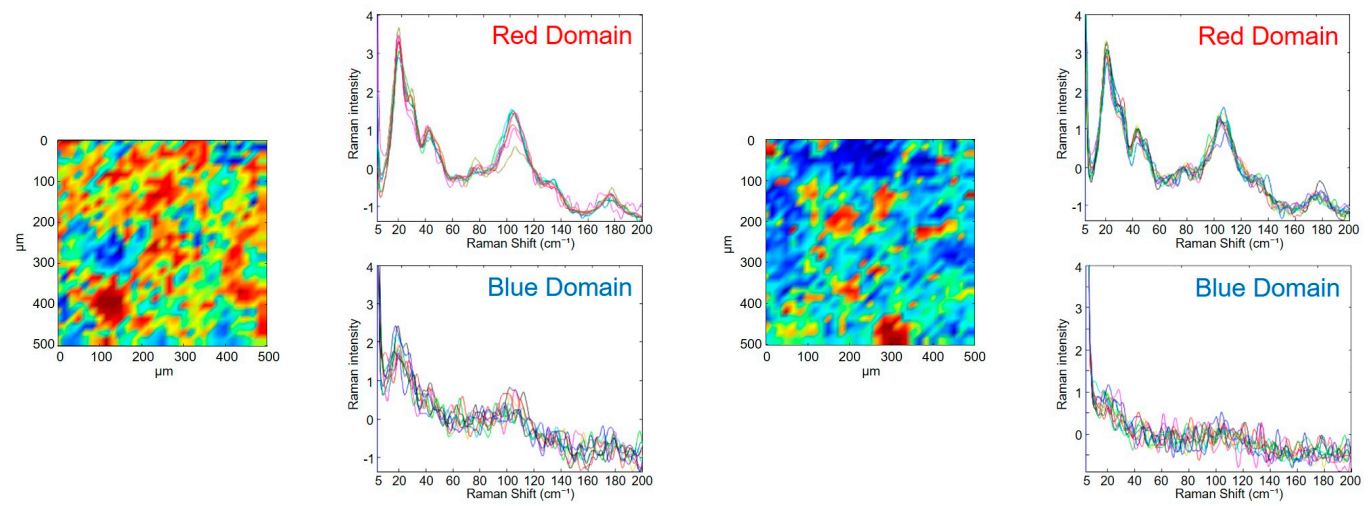

(c)

Figure 3. Low-frequency Raman images and representative spectra of the red domain (upper panels) and blue domain (lower panels) before irradiation (left panels) and after $343 \mathrm{~h}$ of irradiation (right panels): (a) CBZ form III, (b) CBZ-SUC, and (c) CBZ-SAC form II. The Raman spectra of each domain were obtained ten times.

\subsection{Evaluation of Photodegradation on the Surface of Tablets Using FT-IR RAS}

An evaluation of photodegradation of the surface of the sample tablets of CBZ polymorphs, PMs, and cocrystals under a $\mathrm{D}_{65}$ fluorescent lamp was also performed using FT-IR RAS. Since the photodegradation of pharmaceuticals in tablet form is a topochemical reaction, this method is suitable for evaluating the decomposition ratio of a drug on the surface of tablets in a quantitative and reproducible manner $[22,25,26]$. Figure 4 shows a semilogarithmic plot of the degradation profiles of CBZ polymorphs, PMs, and cocrystals. Negative linear relationships were observed between the logarithm of the remaining percentage and irradiation time for each sample, indicating that the degradation of the drug on the surface followed first-order kinetics. Calculated degradation rate constants are shown in Table 2. The degradation rate constants of CBZ polymorphs increased in the order of form III, form I, and form II, consistent with the order of the discoloration rate constants. Degradation rate constants for CBZ-SUC and CBZ-SAC form I were significantly lower than those for the other forms, indicating that the photostability was improved by the formation of these cocrystals. In contrast, the degradation rate constant of CBZ-SAC form II was significantly increased, indicating that CBZ-SAC form II was a photolabile crystal form.

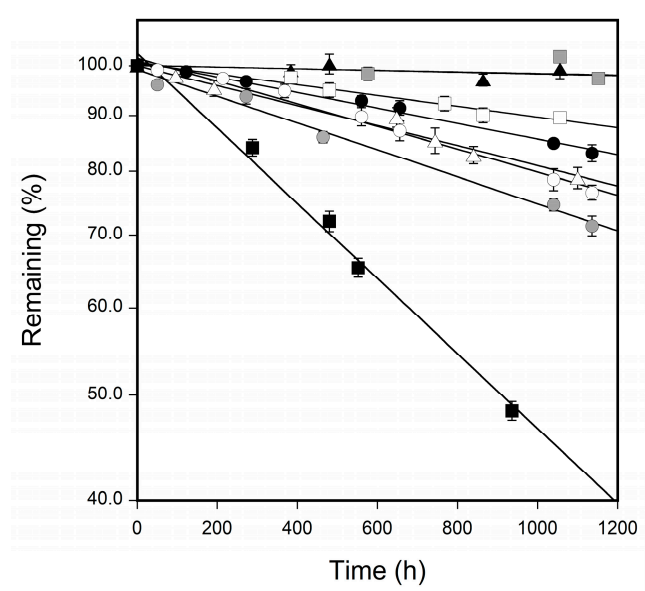

Figure 4. The apparent first-order plots for the photodegradation of CBZ polymorphs, PMs, and cocrystals. The symbols represent changes in the remaining percentages of CBZ form I $(\bigcirc)$, form II (๑),

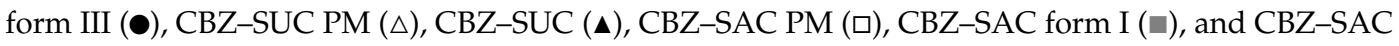
form II (घ). Data are presented as the means \pm SD of 3 values. 
Table 2. Degradation rate constants for CBZ polymorphs, PMs, and cocrystals.

\begin{tabular}{cc}
\hline Sample & Degradation Rate Constant $\left.\mathbf{~ h}^{-\mathbf{1}}\right)$ \\
\hline CBZ form I & $5.53 \times 10^{-4}$ \\
CBZ form II & $6.51 \times 10^{-4}$ \\
CBZ form III & $3.78 \times 10^{-4}$ \\
CBZ-SUC PM & $4.86 \times 10^{-4}$ \\
CBZ-SUC & $3.79 \times 10^{-5}$ \\
CBZ-SAC PM & $2.63 \times 10^{-4}$ \\
CBZ-SAC form I & $4.23 \times 10^{-5}$ \\
CBZ-SAC form II & $1.82 \times 10^{-3}$ \\
\hline
\end{tabular}

\subsection{Investigation of the Relationship between Molecular Mobility and Photostability}

Spin-lattice relaxation times $\left(\mathrm{T}_{1}\right)$ of CBZ polymorphs and cocrystals were measured using ${ }^{1} \mathrm{H}$ SSNMR to investigate the relationship between molecular mobility and photostability. The spin-lattice relaxation time reflects the degree of order and mobility, i.e., the dynamics, in the molecular system $[27,28]$. Figure 5 shows the relationship between ${ }^{1} \mathrm{H} \mathrm{T}_{1}$ values and degradation rate constants. The ${ }^{1} \mathrm{H} \mathrm{T}_{1}$ value of each polymorph was in the order of form II, form I, and form III, that is, the more photostable crystal form had a larger ${ }^{1} \mathrm{H} \mathrm{T}_{1}$ value. A negative linear correlation was observed between the logarithm of ${ }^{1} \mathrm{H} \mathrm{T}_{1}$ and degradation rate constant. Thus, the molecular mobility was significantly decreased by the formation of CBZ-SUC and CBZ-SAC form I, leading to higher photostability.

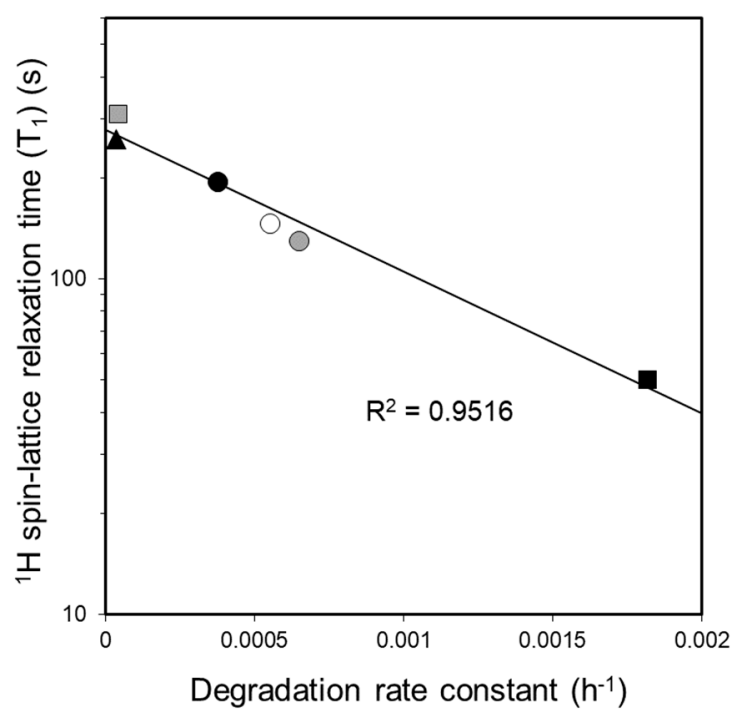

Figure 5. The relationship between the ${ }^{1} \mathrm{H}$ spin-lattice relaxation time $\left(\mathrm{T}_{1}\right)$ and degradation rate

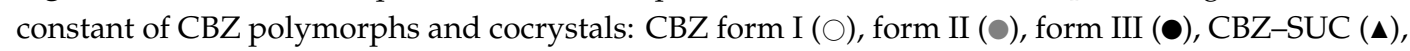
CBZ-SAC form I ( $\square)$, and CBZ-SAC form II ( $\mathbf{\square})$

\section{Discussion}

The cocrystallization technique improves the physicochemical properties of an API without modifying its chemical structure. In the present study, we prepared three cocrystals, including two polymorphs, of CBZ and investigated their photostabilities compared with that of the CBZ polymorphs. CBZ-SUC and CBZ-SAC form I showed better photostability than the conventional crystal forms. The order of the photostability of solid forms estimated using FT-IR RAS was almost consistent with the order obtained from the colorimetric measurement (Figure S4, Supplementary Materials). Based on these results, the discoloration on the surface of the tablets was due to the photodecomposition of API. CBZ is transformed into CBZ cyclobutyl dimer and 10,11-epoxide after irradiation by a mercury-vapor lamp [29] and a near-UV fluorescent lamp [22]. These photoproducts 
are also produced by irradiation with a $\mathrm{D}_{65}$ fluorescent lamp, and the surface color of the tablets subsequently changed. The remaining percentages of CBZ form III, CBZ-SUC, and CBZ-SAC form II estimated using FT-IR RAS were approximately $95 \%, 100 \%$, and $80 \%$, respectively, after $343 \mathrm{~h}$ of irradiation. The results obtained using low-frequency Raman spectroscopy showed the same trends as the data obtained using FT-IR RAS. The low-frequency Raman spectroscopy provides complementary information about the photodecomposition of API on the surface of pharmaceutical preparations in a simple and rapid manner.

Molecular mobility correlates with the photodegradation of CBZ. SUC combines with two CBZ molecules through hydrogen bonding [30]. In addition, CBZ-SAC form I forms a homosynthon between two CBZ molecules, hydrogen bonds between SAC molecules and hydrogen bonds between CBZ and SAC [15]. The dimerization of CBZ induced by photoirradiation needs the reorientation of each CBZ molecule, resulting in a satisfying alignment for the reaction. The formation of alternate stacking configurations of CBZ and the corresponding coformers may hinder the approach of CBZ molecules, thus preventing photodimerization, as reported for vitamin K3 by Zhu et al. [17]. CBZ-SAC form II also forms a heterosynthon through hydrogen bonds between CBZ and SAC [15]; however, this polymorphic form exhibited a lower photostability than CBZ itself. According to the thermodynamic study reported by Pagire et al., CBZ-SAC form I is a stable form, CBZ-SAC form II is a metastable form, and these forms are monotropically related polymorphs [31]. In the present study, the ${ }^{1} \mathrm{H} \mathrm{T}_{1}$ value of CBZ-SAC form II was significantly lower than the other crystal forms, suggesting that the molecular mobility was sufficiently high to allow for a photoreaction. This higher reactivity and instability of CBZ-SAC form II was supported by the findings from previous reports that CBZ-SAC form II is easily converted to form I in a slurry at room temperature $[15,31]$.

In summary, CBZ-SUC and CBZ-SAC form I are photostable crystal forms, and cocrystallization was confirmed to be an effective approach to improving the photostability of a photolabile drug in the present study.

Supplementary Materials: The following are available online at http:/www.mdpi.com/2073-4352/9/11/553/s1, Figure S1: Powder X-Ray Diffractometry (PXRD) patterns of CBZ polymorphs, coformers, PMs, and cocrystals; Figure S2: Differential scanning calorimetry (DSC) profiles of CBZ polymorphs, coformers, PMs, and cocrystals; Figure S3: Standard low-frequency Raman spectra of CBZ form III, CBZ-SUC, CBZ-SAC form II, and microcrystalline cellulose; Figure S4: The relationship between the discoloration rate constant and degradation rate constant.

Author Contributions: Conceptualization, R.T. and T.F.; formal analysis, R.Y. and R.T.; investigation, R.Y., R.H., and C.T.; writing of original draft preparation, R.Y. and R.H.; writing of review and editing, R.T., C.T., T.K., and T.F.; visualization, R.Y. and R.H.; supervision, S.K., T.S., and T.F.; project administration, R.T. and T.F.

Funding: This work was supported in part by JSPS KAKENHI, a Grant-in-Aid for the Scientific Research (C), (grant number: 17K08253) (to T.F.).

Acknowledgments: The authors would like to thank Ryota Hamada for providing technical assistance regarding the experiments.

Conflicts of Interest: The authors declare no conflicts of interest.

\section{References}

1. Coelho, L.; Almeida, I.F.; Sousa Lobo, J.M.; Sousa e Silva, J.P. Photostabilization strategies of photosensitive drugs. Int. J. Pharm. 2018, 541, 19-25. [CrossRef]

2. Janga, K.Y.; King, T.; Ji, N.; Sarabu, S.; Shadambikar, G.; Sawant, S.; Xu, P.; Repka, M.A.; Murthy, S.N. Photostability issues in pharmaceutical dosage forms and photostabilization. AAPS Pharm. Sci. Tech. 2018, 19, 48-59. [CrossRef]

3. Tønnesen, H.H. Formulation and stability testing of photolabile drugs. Int. J. Pharm. 2001, 225, 1-14. [CrossRef]

4. Grzesiak, A.L.; Lang, M.; Kim, K.; Matzger, A.J. Comparison of the four anhydrous polymorphs of carbamazepine and the crystal structure of form I. J. Pharm. Sci. 2003, 92, 2260-2271. [CrossRef] [PubMed]

5. Harris, R.K.; Ghi, P.Y.; Puschmann, H.; Apperley, D.C.; Griesser, U.J.; Hammond, R.B.; Ma, C.; Roberts, K.J.; Pearce, G.J.; Yates, J.R.; et al. Structural studies of the polymorphs of carbamazepine, its dihydrate, and two solvates. Org. Process Res. Dev. 2005, 9, 902-910. [CrossRef] 
6. McMahon, L.E.; Timmins, P.; Williams, A.C.; York, P. Characterization of dihydrates prepared from carbamazepine polymorphs. J. Pharm. Sci. 1996, 85, 1064-1069. [CrossRef]

7. Lowes, M.M.J.; Caira, M.R.; Lotter, A.P.; Van Watt, J.G.D. Physicochemical properties and x-ray structural studies of the trigonal polymorph of carbamazepine. J. Pharm. Sci. 1987, 76, 744-752. [CrossRef]

8. Kobayashi, Y.; Ito, S.; Itai, S.; Yamamoto, K. Physicochemical properties and bioavailability of carbamazepine polymorphs and dihydrate. Int. J. Pharm. 2000, 193, 137-146. [CrossRef]

9. Aakeröy, C.B.; Salmon, D.J. Building co-crystals with molecular sense and supramolecular sensibility. Cryst. Eng. Comm. 2005, 7, 439-448. [CrossRef]

10. Qiao, N.; Li, M.; Schlindwein, W.; Malek, N.; Davies, A.; Trappitt, G. Pharmaceutical cocrystals: An overview. Int. J. Pharm. 2011, 419, 1-11. [CrossRef]

11. Aakeröy, C.B.; Fasulo, M.E.; Desper, J. Cocrystal or salt: Does it really matter? Mol. Pharm. 2007, 4, 317-322. [CrossRef]

12. Childs, S.L.; Rodríguez-Hornedo, N.; Reddy, L.S.; Jayasankar, A.; Maheshwari, C.; McCausland, L.; Shipplett, R.; Stahly, B.C. Screening strategies based on solubility and solution composition generate pharmaceutically acceptable cocrystals of carbamazepine. Cryst. Eng. Comm. 2008, 10, 856-864. [CrossRef]

13. Ullah, M.; Hussain, I.; Sun, C.C. The development of carbamazepine-succinic acid cocrystal tablet formulations with improved in vitro and in vivo performance. Drug Dev. Ind. Pharm. 2016, 42, 969-976. [CrossRef] [PubMed]

14. Hickey, M.B.; Peterson, M.L.; Scoppettuolo, L.A.; Morrisette, S.L.; Vetter, A.; Guzmán, H.; Remenar, J.F.; Zhang, Z.; Tawa, M.D.; Haley, S.; et al. Performance comparison of a co-crystal of carbamazepine with marketed product. Eur. J. Pharm. Biopharm. 2007, 67, 112-119. [CrossRef] [PubMed]

15. Porter, W.W., III; Elie, S.C.; Matzger, A.J. Polymorphism in carbamazepine cocrystals. Cryst. Growth Des. 2008, 8, 14-16. [CrossRef]

16. Wang, J.-R.; Zhou, C.; Yu, X.; Mei, X. Stabilizing vitamin $\mathrm{D}_{3}$ by conformationally selective co-crystallization. Chem. Commun. 2014, 50, 855-858. [CrossRef]

17. Zhu, B.; Wang, J.-R.; Zhang, Q.; Mei, X. Improving dissolution and photostability of vitamin K3 via cocrystallization with naphthoic acids and sulfamerazine. Cryst. Growth Des. 2016, 16, 483-492. [CrossRef]

18. Yu, Q.; Yan, Z.; Bao, J.; Wang, J.-R.; Mei, X. Taming photo-induced oxidation degradation of dihydropyridine drugs through cocrystallization. Chem. Commun. 2017, 53, 12266-12269. [CrossRef]

19. Shinozaki, T.; Ono, M.; Higashi, K.; Moribe, K. A novel drug-drug cocrystal of levofloxacin and metacetamol: Reduced hygroscopicity and improved photostability of levofloxacin. J. Pharm. Sci. 2019, 108, 2383-2390. [CrossRef]

20. Kaneniwa, N.; Yamaguchi, T.; Watari, N.; Otsuka, M. Hygroscopicity of carbamazepine crystalline powders. Yakugaku Zasshi 1984, 104, 184-190. [CrossRef]

21. Matsuda, Y.; Teraoka, R.; Sugimoto, I. Comparative evaluation of photostability of solid-state nifedipine under ordinary and intensive light irradiation conditions. Int. J. Pharm. 1989, 54, 211-221. [CrossRef]

22. Matsuda, Y.; Akazawa, R.; Teraoka, R.; Otsuka, M. Pharmaceutical evaluation of carbamazepine modifications: Comparative study for photostability of carbamazepine polymorphs by using Fourier-transformed reflection-absorption infrared spectroscopy and colorimetric measurement. J. Pharm. Pharmacol. 1994, 46, 162-167. [CrossRef] [PubMed]

23. Koide, T.; Fukami, T.; Hisada, H.; Inoue, M.; Carriere, J.; Heyler, R.; Katori, N.; Okuda, H.; Goda, Y. Identification of pseudopolymorphism of magnesium stearate by using low-frequency Raman spectroscopy. Org. Process. Res. Dev. 2016, 20, 1906-1910. [CrossRef]

24. Walker, G.; Römann, P.; Poller, B.; Löbmann, K.; Grohganz, H.; Rooney, J.S.; Huff, G.S.; Smith, G.P.S.; Rades, T.; Gordon, K.C.; et al. Probing pharmaceutical mixtures during milling: The potency of low-frequency Raman spectroscopy in identifying disorder. Mol. Pharm. 2017, 14, 4675-4684. [CrossRef]

25. Teraoka, R.; Otsuka, M.; Matsuda, Y. Evaluation of photostability of solid-state dimethyl 1,4-dihydro-2,6-dimethyl-4-(2-nitro-phenyl)-3, 5-pyridinedicarboxylate by using Fourier-transformed reflection-absorption infrared spectroscopy. Int. J. Pharm. 1999, 184, 35-43. [CrossRef]

26. Teraoka, R.; Otsuka, M.; Matsuda, Y. Evaluation of photostability of solid-state nicardipine hydrochloride polymorphs by using Fourier-transformed reflection-absorption infrared spectroscopy-Effect of grinding on the photostability of crystal form. Int. J. Pharm. 2004, 286, 1-8. [CrossRef] 
27. Lubach, J.W.; Xu, D.; Segmuller, B.E.; Munson, E.J. Investigation of the effects of pharmaceutical processing upon solid-state NMR relaxation times and implications to solid-state formulation stability. J. Pharm. Sci. 2007, 96, 777-787. [CrossRef]

28. Hudson, S.P.; Owens, E.; Hughes, H.; McLoughlin, P. Enhancement and restriction of chain motion in polymer networks. Int. J. Pharm. 2012, 430, 34-41. [CrossRef]

29. Robson, J.K.; Sharples, D. Photoirradiation products of cyproheptadine and carbamazepine. J. Pharm. Pharmacol. 1984, 36, 843-844. [CrossRef]

30. Shenmin, X.; Dang, L.; Wei, H. Solid-liquid phase equilibrium and phase diagram for the ternary carbamazepinesuccinic acid-ethanol or acetone system at (298.15 and 308.15) K. J. Chem. Eng. Data 2011, 56, 2746-2750. [CrossRef]

31. Pagire, S.K.; Jadav, N.; Vangala, V.R.; Whiteside, B.; Paradkar, A. Thermodynamic investigation of carbamazepine-saccharin co-crystal polymorphs. J. Pharm. Sci. 2017, 106, 2009-2014. [CrossRef] [PubMed]

C 2019 by the authors. Licensee MDPI, Basel, Switzerland. This article is an open access article distributed under the terms and conditions of the Creative Commons Attribution (CC BY) license (http://creativecommons.org/licenses/by/4.0/). 